\title{
New Mathematical Models of Compact Stars with Charge Distributions
}

\author{
Manuel Malaver \\ Department of Basic Sciences, Maritime University of the Caribbean, Catia la Mar, Venezuela \\ Email address: \\ mmf.umc@gmail.com
}

\section{To cite this article:}

Manuel Malaver. New Mathematical Models of Compact Stars with Charge Distributions. International Journal of Systems Science and Applied Mathematics. Vol. 2, No. 5, 2017, pp. 93-98. doi: 10.11648/j.ijssam.20170205.13

Received: August 23, 2017; Accepted: September 19, 2017; Published: October 23, 2017

\begin{abstract}
In this paper, have been found new class of solutions to the Einstein-Maxwell system for charged anisotropic matter which are relevant in the description of highly compact stellar objects. The equation of state is barotropic with a linear relation between the radial pressure and the energy density and we have considered a prescribed form for the gravitational potential Z. Variables as the energy density, radial pressure and the metric coefficients are written in terms of elementary and polynomial functions. The obtained models not admit singularities in the matter and the charge density.
\end{abstract}

Keywords: Einstein-Maxwell System, Charged Anisotropic Matter, Compact Stellar Objects, Energy Density, Metric Coefficients

\section{Introduction}

From the development of Einstein's theory of general relativity, the modelling of superdense mater configurations is an interesting research area $[1,2]$. In the last decades, such models allow explain the behavior of massive objects as neutron stars, quasars, pulsars, black holes and white dwarfs $[3,4,5]$. Malaver [3] studied the behavior of the thermal capacity $\mathrm{C}_{\mathrm{v}}$ for Schwarzschild's black hole when $\mathrm{T}>>\mathrm{T}_{\mathrm{C}}$ and $\mathrm{T}<<\mathrm{T}_{\mathrm{C}}$ where $\mathrm{T}_{\mathrm{C}}$ is the characteristic temperature of the Schwarzschild black hole and found that the value for $\mathrm{C}_{\mathrm{v}}$ if $\mathrm{T}>>\mathrm{T}_{\mathrm{C}}$ is the same that would be obtained in an ideal diatomic gas if only are considered the degrees of freedom rotational. Komathiraj and Maharaj [4] find new classes exact solutions to the Einstein-Maxwell system of equations for a charged sphere with a particular choice of the electric field intensity and one of the gravitational potentials. Sharma et al. [5] have obtained a class of solutions to the EinsteinMaxwell system assuming a particular form for the hypersurface $(\mathrm{t}=$ constant $)$ containing a parameter $\lambda$.

In theoretical works of realistic stellar models, is important include the pressure anisotropy [6-8]. The existence of anisotropy within a star can be explained by the presence of a solid core, phase transitions, a type III super fluid, a pion condensation [9] or another physical phenomenon as the presence of the electrical field [10]. The physics of ultrahigh densities is not well understood and many of the strange stars studies have been performed within the framework of the MIT-Bag model [11]. In this model, the strange matter equation of state has a simple linear form given by $p=\frac{1}{3}(\rho-4 B)$ where $\rho$ is the energy density, $\mathrm{p}$ is the isotropic pressure and $\mathrm{B}$ is the bag constant. Many researchers have used a great variety of mathematical techniques to try to obtain exact solutions for quark stars within the framework of MIT-Bag model; Komathiraj and Maharaj [11] found two new classes of exact solutions to the Einstein-Maxwell system of equations with a particular form of the gravitational potential and isotropic pressure. Malaver $[12,13]$ also has obtained some models for quark stars considering a potential gravitational that depends on an adjustable parameter. Thirukkanesh and Maharaj [14] studied the behavior of compact relativistic objects with anisotropic pressure in the presence of the electromagnetic field. Maharaj et al. [15] generated new models for quark stars with charged anisotropic matter considering a linear equation of state. Thirukkanesh and Ragel [16] obtained new models for compact stars with quark matter. Sunzu et al. found new classes of solutions with specific forms for the measure of anisotropy [17].

With then use of Einstein's field equations, important advances has been made to model the interior of a star. In particular, Feroze and Siddiqui [18, 19] and Malaver [20-23] 
consider a quadratic equation of state for the matter distribution and specify particular forms for the gravitational potential and electric field intensity. Mafa Takisa and Maharaj [24] obtained new exact solutions to the EinsteinMaxwell system of equations with a polytropic equation of state. Thirukkanesh and Ragel [25] have obtained particular models of anisotropic fluids with polytropic equation of state which are consistent with the reported experimental observations. Malaver [26, 27] generated new exact solutions to the Einstein-Maxwell system considering Van der Waals modified equation of state with and without polytropical exponent and [28] presented a anisotropic strange quark matter model by imposing a linear barotropic equation of state with Tolman IV form for the gravitational potential. Mak and Harko [29] found a relativistic model of strange quark star with the suppositions of spherical symmetry and conformal Killing vector. Bhar et al. [30, 31] have studied extensively the behavior of static spherically symmetric relativistic objects with anisotropic matter distribution considering the Tolman VII space-time. Pant et al. [32] have found new exact solutions of the field equations for anisotropic neutral fluid in isotropic coordinates.

The objective of this paper is to obtain new exact solutions to the Maxwell-Einstein system for charged anisotropic matter with lineal equation of state in static spherically symmetric spacetime using a prescribed form for the gravitational potential Z. This article is organized as follows, in Section 2, presents Einstein's field equations of anisotropic fluid distribution. In Section 3, is chosen a particular choice of gravitational potential $\mathrm{Z}(\mathrm{x})$ that allows solving the field equations and are obtained new models for charged anisotropic matter. In Section 4, a physical analysis of the new solution is performed. Finally in Section 5, we conclude.

\section{Einstein Field Equations}

We consider a spherically symmetric, static and homogeneous and anisotropic spacetime in Schwarzschild coordinates given by

$$
d s^{2}=-e^{2 v(r)} d t^{2}+e^{2 \lambda(r)} d r^{2}+r^{2}\left(d \theta^{2}+\sin ^{2} \theta d \varphi^{2}\right)
$$

where $v(r)$ and $\lambda(r)$ are two arbitrary functions.

The Einstein field equations for the charged anisotropic matter are given by

$$
\begin{aligned}
& T_{00}=-\rho-\frac{1}{2} E^{2} \\
& T_{11}=p_{r}-\frac{1}{2} E^{2} \\
& T_{22}=T_{33}=p_{t}+\frac{1}{2} E^{2}
\end{aligned}
$$

where $\rho$ is the energy density, $p_{r}$ is the radial pressure, $E$ is electric field intensity and $p_{t}$ is the tangential pressure, respectively. Using the transformations, $x=c r^{2}, Z(x)=e^{-2 \lambda(r)}$ and $A^{2} y^{2}(x)=e^{2 v(r)}$ with arbitrary constants A and $c>0$, suggested by Durgapal and Bannerji [33], the Einstein field equations can be written as

$$
\begin{gathered}
\frac{1-Z}{x}-2 \dot{Z}=\frac{\rho}{c}+\frac{E^{2}}{2 c} \\
4 Z \frac{\dot{y}}{y}-\frac{1-Z}{x}=\frac{p_{r}}{c}-\frac{E^{2}}{2 c} \\
4 x Z \frac{\ddot{y}}{y}+(4 Z+2 \times \dot{Z}) \frac{\dot{y}}{y}+\dot{Z}=\frac{p_{t}}{c}+\frac{E^{2}}{2 c} \\
\frac{\Delta}{c}=4 x Z \frac{\ddot{y}}{y}+\dot{Z}\left(1+2 x \frac{\dot{y}}{y}\right)+\frac{1-Z}{x}-\frac{E^{2}}{c} \\
\sigma_{t}^{2}=\frac{4 c Z}{x}(x \dot{E}+E)^{2}
\end{gathered}
$$

$\sigma$ is the charge density, $\Delta=p_{t}-p_{r}$ is the anisotropic factor and dots denote differentiation with respect to $\mathrm{x}$. With the transformations of [33], the mass within a radius $r$ of the sphere take the form

$$
M(x)=\frac{1}{4 c^{3 / 2}} \int_{0}^{x} \sqrt{x}\left(\rho_{*}+E^{2}\right) d x
$$

where $\rho_{*}=\left(\frac{1-Z}{x}-2 \dot{Z}\right) c$

In this paper, we assume the following lineal equation of state within the framework of MIT-Bag model

$$
p_{r}=\frac{1}{3} \rho
$$

\section{Classes of Models}

In agreement with Feroze and Siddiqui [18] and Malaver [34], is chosen the form of the gravitational potential, $Z(x)$ as

$$
Z(x)=\left(\frac{1-a x}{1+a x}\right)^{n}
$$

where $\mathrm{a}$ is real constant and $\mathrm{n}$ an adjustable parameter. This potential is well behaved and regular at the origin in the interior of the sphere. In this work have been considered the particular form of the electrical field proposed for Malaver [23]

$$
E^{2}=2 c(1-Z)
$$


For the case $\mathrm{n}=1$, using $Z(x)$ and eq. (14) in eq. (5) we obtain

$$
\rho=\frac{c\left[6 a+2 a(a-1) x-2 a^{2} x^{2}\right]}{(1+2 a x)^{2}}
$$

Substituting (15) in eq. (12), the radial pressure can be written in the form

$$
p_{r}=\frac{c\left[6 a+2 a(a-1) x-2 a^{2} x^{2}\right]}{3(1+2 a x)^{2}}
$$

Replacing (15) in eq. (11) we have for the mass function

$$
M(x)=\frac{x^{1 / 2}}{\sqrt{c}}\left[\frac{\left(3 a^{2} x-a^{2} x^{2}+2 a x+3\right)}{3 a(1+a x)}\right]-\frac{\arctan (\sqrt{a x})}{a \sqrt{a c}}
$$

With (13) in eq. (14) we obtain for the electric field intensity

$$
E^{2}=\frac{4 a c x}{1+a x}
$$

and for charge density

$$
\sigma^{2}=\frac{4 a c^{2}(1-a x)(3+2 a x)^{2}}{(1+a x)^{4}}
$$

$$
\frac{\dot{y}}{y}=\frac{6 a+2 a(a-1) x-2 a^{2} x^{2}}{12(1+a x)(1-a x)}+\frac{a(1-x)}{2(1-a x)}
$$

Integrating (20) it is obtained

$$
y(x)=c_{1}(1+a x)^{1 / 6}(-1+a x)^{\frac{4-5 a}{6 a}} e^{\frac{2 x}{3}}
$$

$c_{1}$ is the constant of integration.

The metric functions $e^{2 \lambda}$ and $e^{2 v}$ can be written as

$$
e^{2 \lambda}=\frac{1+a x}{1-a x}
$$

$$
e^{2 v}=A^{2} c_{1}^{2}(1+a x)^{1 / 3}(-1+a x)^{\frac{4-5 a}{3 a}} e^{\frac{4 x}{3}}
$$

and the anisotropy factor can be written as

$$
\begin{aligned}
& \Delta=\frac{4 c x}{9}\left[\begin{array}{l}
-\frac{5 a^{2}(1-a x)}{4(1+a x)^{3}}+\frac{45 a^{2}-26 a-4 a x-4}{(1+a x)}+\frac{16-64 a+55 a^{2}}{4(1-a x)(1+a x)} \\
+\frac{2 a(1-a x)}{(1+a x)^{2}}
\end{array}\right] \\
& -\frac{2 c\left[\left(4 a^{3}+4 a^{2}-5 a^{4}\right) x^{2}+\left(4 a+8 a^{2}-5 a^{3}\right) x+3 a\right]}{3(1+a x)^{3}}+\frac{2 a c(1-2 x)}{1+a x}
\end{aligned}
$$

The metric for this model is

Substituting (13), (14) and (16) in (6), we have

$$
d s^{2}=-A^{2} c_{1}^{2}\left(1+a r^{2}\right)^{1 / 3}\left(-1+a r^{2}\right)^{\frac{4-5 a}{3 a}} e^{\frac{4 r^{2}}{3}} d t^{2}+\frac{\left(1+a r^{2}\right)}{\left(1-a r^{2}\right)} d r^{2}+r^{2}\left(d \theta^{2}+\sin ^{2} \theta d \phi^{2}\right)
$$

With $n=2$, we can find the following analytical model

$$
\begin{gathered}
\rho=\frac{c\left[12 a-4 a(a+1) x-4 a^{2} x^{2}\right]}{(1+2 a x)^{3}} \\
p_{r}=\frac{c\left[12 a-4 a(a+1) x-4 a^{2} x^{2}\right]}{3(1+2 a x)^{3}} \\
M(x)=\frac{2 x^{1 / 2}}{\sqrt{c}}\left[\frac{2 a-a x-1}{a(1+a x)}\right]+\frac{2(a+1) \arctan (\sqrt{a x})}{a \sqrt{a c}} \\
E^{2}=\frac{8 a c x}{(1+a x)^{2}}
\end{gathered}
$$

and $c_{2}$ is the constant of integration.

$$
\begin{aligned}
& \Delta=\frac{4 c x(1-a x)^{2}}{9(1+a x)^{2}}\left[\begin{array}{l}
-\frac{2 a^{2}}{(1+a x)^{2}}+\frac{2 a(4+a)}{(1+a x)(1-a x)}+\frac{8 a(a-1)}{(1+a x)(a x-1)^{2}} \\
+\frac{10 a^{2}+20 a+16}{(a x-1)^{2}}-\frac{32\left(a^{2}-1\right)}{(a x-1)^{3}}+\frac{16\left(a^{2}-1\right)}{(a x-1)^{4}}
\end{array}\right] \\
& -\frac{4 a(1-a x)(5 a x+3)}{3(1+a x)^{4}}-\frac{8 a x(4+a)}{3(1+a x)^{3}}-\frac{32 a x(a-1)}{3(1+a x)^{3}(1-a x)}+\frac{4 a(1-2 x)}{(1+a x)^{2}}
\end{aligned}
$$


The metric for this model is

$$
d s^{2}=-A^{2} c_{2}^{2}\left(1+a r^{2}\right)^{2 / 3}\left(-1+a r^{2}\right)^{-\frac{2(4+a)}{3 a}} e^{-\frac{8(a-1)}{3 a\left(a r^{2}-1\right)}} d t^{2}+\frac{\left(1+a r^{2}\right)^{2}}{\left(1-a r^{2}\right)^{2}} d r^{2}+r^{2}\left(d \theta^{2}+\sin ^{2} \theta d \phi^{2}\right)
$$

\section{Physical Features of the New Models}

Any physically acceptable solutions must satisfy the following conditions [25]:

(1) Regularity of the gravitational potentials in the origin.

(2) Radial pressure must be finite at the centre.

(3) $P_{r}>0$ and $\rho>0$ in the origin.

(4) Monotonic decrease of the energy density and the radial pressure with increasing radius

With $n=1 \quad e^{2 \lambda(0)}=1 \quad, \quad e^{2 v(0)}=A^{2} c_{1}^{2}(-1)^{\frac{4-5 a}{3 a}}$ and $\left(e^{2 \lambda(r)}\right)_{r=0}^{\prime}=\left(e^{2 v(r)}\right)_{r=0}^{\prime}=0$ This demonstrates that the gravitational potential is regular in the origin. At the centre $r=0, \rho(0)=6 a c, p_{r}(0)=2 a c$ both are positive if a $>0$. In the surface of the star $r=R$ we have $p_{r}(R)=0$ and
$R=\frac{\sqrt{2 a c\left(a-1+\sqrt{a^{2}+10 a+1}\right)}}{2 a c}$. This is a new value found for the radius of the star. In this case it is obtained

$$
\begin{aligned}
& \frac{d \rho}{d r}=\frac{4 a c^{2}(a-1) r-8 a^{2} c^{3} r^{3}}{\left(2 a c r^{2}+1\right)^{2}}-\frac{8 a c r\left(6 a c+2 a c^{2}(a-1) r^{2}-2 a^{2} c^{3} r^{4}\right)}{\left(2 a c r^{2}+1\right)^{3}} \\
< & 0 \text { and } \\
& \frac{d p_{r}}{d r}=\frac{4 a c^{3}(a-1) r-8 a^{2} c^{3} r^{3}}{3\left(2 a c r^{2}+1\right)^{2}}-\frac{8 a c r\left(6 a c+2 a c^{3}(a-1) r^{2}-2 a^{2} c^{3} r^{4}\right)}{3\left(2 a c r^{2}+1\right)^{3}}
\end{aligned}
$$

$<0$. The energy density and radial pressure decrease from the centre to the surface of the star.

From (17), it is deduced

$$
M(r)=\frac{\sqrt{c r^{2}}}{\sqrt{c}}\left[\frac{\left(3 a^{2} c r^{2}-a^{2} c^{2} r^{4}+2 a c r^{2}+3\right)}{3 a\left(1+a c r^{2}\right)}\right]-\frac{\arctan \left(\sqrt{a c r^{2}}\right)}{a \sqrt{a c}}
$$

$$
\begin{aligned}
& M(r=R)=\frac{\sqrt{2 a c\left(a-1+\sqrt{a^{2}}+10 a+1\right)}}{2 a c}\left(\frac{\left(3 a^{2}+2 a\right) c\left(\frac{2 a c\left(a-1+\sqrt{a^{2}+10 a+1}\right)}{2 a c}\right)}{3 a\left(1+a c\left[\frac{a-1+\sqrt{a^{2}+10 a+1}}{2 a c}\right]\right)}\right] \\
& -\frac{\arctan \left(\frac{\sqrt{2 a c\left(a-1+\sqrt{a^{2}+10 a+1}\right)}}{2 a c} \sqrt{a c}\right)}{a \sqrt{a c}}
\end{aligned}
$$

For the case $n=2 \quad e^{2 \lambda(0)}=1$, $e^{2 v(0)}=A^{2} c_{1}^{2}(-1)^{-\frac{2(4+a)}{3 a}} e^{\frac{8(a-1)}{3 a}}$ in the origin and $\left(e^{2 \lambda(r)}\right)_{r=0}^{\prime}=\left(e^{2 v(r)}\right)_{r=0}^{\prime}=0$. Again the gravitational potential is regular in $r=0$. In the centre $r=0$ $\rho(0)=12 a c$ and $p_{r}(0)=4 a c$, are both are positive if a $>0$. In the boundary of the star $r=R$ we have $p_{r}(r=R)=0$ and

$$
R=\frac{\sqrt{2 a c\left(a c+c+\sqrt{a^{2} c^{2}+2 a c^{2}+12 a c+c^{2}}\right)}}{2 a c} \text {. For this model we }
$$
have 


$$
\begin{aligned}
\quad \frac{d \rho}{d r} & =-\frac{8 a c^{2}(a-1) r-16 a^{2} c^{3} r^{3}}{\left(2 a c r^{2}+1\right)^{3}}-\frac{12 a c r\left(12 a c-4 a c^{2}(a-1) r^{2}-4 a^{2} c^{3} r^{4}\right)}{\left(2 a c r^{2}+1\right)^{4}} \\
<0 & \text { and } \\
\frac{d p_{r}}{d r} & =-\frac{8 a c^{2}(a-1) r-16 a^{2} c^{3} r^{3}}{3\left(2 a c r^{2}+1\right)^{3}}-\frac{4 a c r\left(12 a c-4 a c^{2}(a-1) r^{2}-4 a^{2} c^{3} r^{4}\right)}{\left(2 a c r^{2}+1\right)^{4}}<
\end{aligned}
$$

0 for all $0<\mathrm{r}<\mathrm{R}$. The energy density and radial pressure decrease from the centre to the surface of the star.

From (28) we have

$$
M(r)=2 r\left[\frac{2 a-a c r^{2}-1}{a\left(1+a c r^{2}\right)}\right]+\frac{2(a+1) \arctan (r \sqrt{a c})}{a \sqrt{a c}}
$$

and the total mass of the star is

$$
\begin{aligned}
& M(r)=\frac{\sqrt{2 a c\left(a c+c+\sqrt{a^{2} c^{2}+2 a c^{2}+12 a c+c^{2}}\right)}}{a c} \\
& {\left[\frac{2 a-\frac{\left(a c+c+\sqrt{a^{2} c^{2}+2 a c^{2}+12 a c+c^{2}}\right)}{2}-1}{a\left(1+\frac{\left(a c+c+\sqrt{a^{2} c^{2}+2 a c^{2}+12 a c+c^{2}}\right)}{2}\right)}\right]+} \\
& 2(a+1) \arctan \left(\frac{\sqrt{2 a c\left(a c+c+\sqrt{a^{2} c^{2}+2 a c^{2}+12 a c+c^{2}}\right)}}{2 a c} \sqrt{a c}\right. \\
& \left.\frac{a \sqrt{a c}}{2}\right)
\end{aligned}
$$

\section{Conclusion}

In this paper, we have generated new exact solutions to the Einstein-Maxwell system considering a prescribed form for the gravitational potential $\mathrm{Z}$ and a lineal equation of state within the framework of MIT-Bag model which is very useful for realistic models of stars. The new model satisfy the system of equations (6) - (9) and constitute another new family of solutions for anisotropic charged matter. The metric functions $e^{2 \lambda}$ and $e^{2 v}$ can be written in terms of elementary functions and the variables energy density, pressure and charge density also are represented analytical. The mass function is continuous and behaves well inside of the star and the charge density $\sigma$ not admits singularities at the center. The radial pressure, energy density and the coefficients of the metric are well defined. The charge density $\sigma$ not admits singularities at the centre of the stellar object and the mass function is an increasing function, continuous and finite. The gravitational potentials are regular at the centre and well behaved. The relativistic solution to the Einstein-Maxwell system presented are physically reasonable. The new obtained model may be used to model relativistic charged compact objects in different astrophysical scenes as quark and neutron stars.

\section{References}

[1] P. K. Kuhfitting, Some remarks on exact wormhole solutions, Adv. Stud. Theor. Phys., 5, 365- 367, 2011.

[2] J. Bicak, Einstein equations: exact solutions, Encyclopedia of Mathematical Physics, 2, 165-173, 2006.

[3] M. Malaver, Black Holes, Wormholes and Dark Energy Stars in General Relativity. Lambert Academic Publishing, Berlin. ISBN: 978-3-659-34784-9, 2013.

[4] K. Komathiraj, S. D. Maharaj, Classes of exact EinsteinMaxwell solutions, Gen. Rel. Grav., 39, 2079-2093, 2008.

[5] R. Sharma, S, Mukherjee, S. D. Maharaj, General solution for a class of static charged stars, Gen. Rel. Grav., 33, 999-110, 2001.

[6] R. L. Bowers, E. P. T. Liang, Anisotropic spheres in general relativity, Astrophys. J., 188, 657, 1974.

[7] M. Cosenza, L. Herrera, M. Esculpi, L. Witten, Some models of anisotropic spheres in general relativity, J. Math. Phys., 22(1), 118, 1981.

[8] M. K. Gokhroo, A. L. Mehra, Anisotropic spheres with variable energy density in general relativity, Gen. Relat. Grav., 26(1), 75-84, 1994.

[9] A. I. Sokolov, Phase transitions in a superfluid neutron liquid, Sov. Phys. JETP., 52, 575, 1980.

[10] V. V. Usov, Electric fields at the quark surface of strange stars in the color-flavor locked phase, Phys. Rev. D, 70, 067301, 2004.

[11] K. Komathiraj, S. D. Maharaj, Analytical models for quark stars, Int. J. Mod. Phys., D16, pp. 1803-1811, 2007.

[12] M. Malaver, Models for quark stars with charged anisotropic matter, Research Journal of Modeling and Simulation, 1(4), 65-71, 2014.

[13] M. Malaver, Some new models for strange quark stars with isotropic pressure, AASCIT Communications, 1, 48-51, 2014.

[14] S. Thirukkanesh, S. D. Maharaj, Charged anisotropic matter with linear equation of state, Class. Quantum Gravity, 25, $235001,2008$.

[15] S. D. Maharaj, J. M, Sunzu, and S. Ray, Some simple models for quark stars, Eur. Phys. J. Plus., 129, 3, 2014.

[16] S. Thirukkanesh, F. C. Ragel, A class of exact strange quark star model, PRAMANA-Journal of physics, 81(2), 275-286, 2013.

[17] J. M. Sunzu, S. D. Maharaj S. Ray, Quark star model with charged anisotropic matter, Astrophysics. Space. Sci. 354, 517- 524, 2014

[18] T. Feroze, A. Siddiqui, Charged anisotropic matter with quadratic equation of state, Gen. Rel. Grav., 43, 1025-1035, 2011, 2011.

[19] T. Feroze, and A. Siddiqui, Some exact solutions of the Einstein-Maxwellequations with a quadratic equation of state, Journal of the Korean Physical Society, 65(6), 944-947, 2014. 
[20] M. Malaver, Strange quark star model with quadratic equation of state, Frontiers of Mathematics and Its Applications., 1(1), 9-15, 2014.

[21] M. Malaver, Quark star model with charge distributions, Open Science Journal of Modern Physics., 1(1), 6-11, 2014.

[22] M. Malaver, Relativistic Modeling of Quark Stars with Tolman IV Type Potential, International Journal of Modern Physics and Application., 2(1), 1-6, 2015.

[23] M. Malaver, Classes of Relativistic Stars with Quadratic Equation of State, World Scientific News., 57, 70-80, 2016.

[24] P. M. Takisa, S. D. Maharaj, Some charged polytropic models, Gen. Rel. Grav., 45, 1951-1969, 2013.

[25] S. Thirukkanesh, F. C. Ragel, Exact anisotropic sphere with polytropic equation of state, PRAMANA-Journal of physics, 78(5), 687-696, 2012.

[26] M. Malaver, Analytical model for charged polytropic stars with Van der Waals Modified Equation of State, American Journal of f Astronomy and Astrophysics, 1(4), 41-46, 2013.

[27] M. Malaver, Regular model for a quark star with Van der Waals modified equation of state, World Applied Programming., 3, 309-313, 2013.
[28] S. Thirukkanesh, F. C. Ragel, Strange star model with Tolmann IV type potential, Astrophysics and Space Science, 352(2), 743-749, 2014.

[29] M. K Mak, T. Harko, Quark stars admitting a one-parameter group of conformal motions, Int. J. Mod. Phys, D13, 149-156, 2004.

[30] P. Bhar, M. H. Murad, N. Pant, Relativistic anisotropic stellar models with Tolman VII spacetime, Astrophysics and Space Science, 359: 13. doi: 10.1007/s10509-015-2462-9, 2015.

[31] P. Bhar, K. N, Singh, N. Pant, Compact star modeling with quadratic equation of state in Tolman VII spacetime, Indian Journal of Physics. doi: 10.1007/s12648-017-0963-9, 2017.

[32] N. Pant, N. Pradhan, M. Malaver. Anisotropic fluid star model in isotropic coordinates. Int. J. Astrophys. Space. Sci. 3(1), 1$5,2015$.

[33] M. C. Durgapal, R. Bannerji, New analytical stellar model in general relativity, Phys. Rev. D27, 328-331, 1983.

[34] M. Malaver, Analytical models for compact stars with a linear equation of state World Scientific News., 50, 64-73, 2016. 